\title{
LA CONTINUIDAD ONTOLÓGICA EN EL PENSAMIENTO DE PROCLO
}

\author{
THE ONTOLOGICAL CONTINUITY IN THE PROCLUS THOUGHT
}

\author{
Gabriela de los Ángeles Caram ${ }^{1}$ \\ Universidad Nacional de Cuyo - CONICET (Argentina)
}

Recibido: 31-04-2013

Aceptado: 10-10-2013

Resumen: Proclo expone los principios de su reflexión en un orden rigurosamente articulado, manifestando una continuidad latente en la totalidad del universo, e interpretándolo en términos de estricta causalidad, que conecta al Uno con los variados estadíos de ser, dispuestos jerárquicamente en una cadena descendente y sin fisuras entre sus miembros.

El sistema procleano se presenta como una trama dinámica de relaciones que resultan inmediatamente inteligibles, permitiendo estrechar la distancia existente entre los diferentes niveles de la realidad. El propósito de este estudio es apreciar los principales fundamentos de unidad y continuidad que cimentan el pensamiento neoplatónico del autor de Bizancio.

Palabras-clave: Proclo, Neoplatonismo, Jerarquía de seres, Continuidad ontológica.

\begin{abstract}
Proclus explains the principles of his reflection in a rigorously articulated order, manifesting latent continuity in the entire universe, and interpreting it in terms of strict causality, which connects the One with the various stages of being arranged hierarchically in a descending chain without fissures between its members.

Proclean system is seen as a dynamic web of relationships that are immediately intelligible, allowing narrow the gap between the different levels of reality. The purpose of this study is to appraise the main foundations of unity and continuity that support the thinking of the author of Byzantium.
\end{abstract}

Key-words: Proclus, Neoplatonism, Hierarchy of beings, Ontological continuity.

[1] (gabycaram@hotmail.com) Gabriela de los Ángeles Caram es profesora y licenciada en Filosofía, egresada de la Universidad Nacional de Cuyo, e Investigadora Becaria en CONICET (UNCuyo-Argentina). 


\section{Introducción}

Proclo de Bizancio (n. 8 de febrero de 410, en Constantinopla; $\dagger 17$ de abril, de 485 en Atenas), fue discípulo de Plutarco de Atenas y de Siriano. El neoplatonismo con él cobra el carácter de sistema erudito en Atenas, con repercusiones platonizantes en el entorno de la filosofía musulmana y medieval cristiana; aunque la escolástica sólo pudo conocer fragmentariamente a Proclo a partir de 1268, entre algunos discípulos de San Alberto Magno.

Proclo fue el más importante miembro de la escuela del neoplatonismo después de su fundador Plotino. La discrepancia de Jámblico ${ }^{2}$ con la filosofía de Plotino, fue seguida posteriormente por Siriano y Proclo, quienes elaboraron sus propios sistemas de pensamiento. Es cierto que antes de Proclo, la cristiandad había sido profundamente influenciada por las ideas neoplatónicas derivadas de Plotino a través de un intermediario tan importante como San Agustín. Sin embargo, las principales fuentes de neoplatonismo para la escolástica fueron fundamentalmente: Dionisio Pseudo-Areopagita, Proclo y el Liber de Causis, respecto del cual los contemporáneos de Santo Tomás supusieron que se trataba de un trabajo aristotélico.

$\mathrm{Su}$ pensamiento nos hace llegar los principios elementales de la mencionada doctrina neoplatónica, en un estricto orden de conexión lógica, configurando una totalidad correctamente articulada. La característica esencial de la filosofía de este autor consiste en ser un sistema ontológico de la identidad, en el cual la analogía aparece como el fundamento de la estructura unitaria del universo ${ }^{3}$. El sistema procleano no se presenta como un conjunto global estático y rígido, sino que es el realizarse orgánico de una compleja trama de relaciones articuladas de manera dinámica. Así, la identidad en este pensador implica diferenciación y relacionalidad estructural, en cuanto que la identidad se va verificando a través de las relaciones y diferenciaciones continuas.

Su obra más destacada fue Elementos de Teología $a^{4}$, un tratado relativamente corto que comprende dos secciones principales, y uno de los manuales más sistemáticos entre su vasta obra, la cual no se conserva completa ${ }^{5}$. La metafísica procleana es un armónico sistema lógico en el cual se trata de demos-

[2] Jámblico de Calcis (240-325 d.C) fue un filósofo sirio, uno de los principales exponentes del neoplatonismo y discípulo de Porfirio en Roma; luego conoció la filosofía de Plotino, de la cual se separó para regresar a Siria y fundar su propia escuela, que intentaba conciliar, en un sistema único y coherente, las ideas de Platón con las del pensador y matemático Pitágoras, y con algunos elementos místicos de la religión oriental .

[3] La analogía une los extremos del universo en un orden unitario, en el cual todo deviene afín al Uno, para retornar a Él, en virtud de su semejanza.

[4] Proclus, Elementa Theologiae, E. R. Dodds (2nd. ed.), Oxford: Clarendon Press, 1963 (Repr. 1977). Cit. E.T.

[5] Sobre este tema ver Radek Chlup, Proclus. An introduction, Cambridge: Cambridge University Press, 2012, p. 43.

THÉMATA. Revista de Filosofía, Nº49 enero-junio (2014) pp.: 105-125 doi: 10.12795/themata.2014.i49.06 
trar la coherencia formal de sus proposiciones. En efecto, Proclo utiliza el método euclideano ${ }^{6}$ para realizar la derivación de un complejo sistema compuesto por teoremas metafísicos que se fundan en un pequeño número de axiomas iniciales. La estructura lógica que reproduce detalladamente su pensamiento, refleja la estructura lógica de la realidad, cuyos niveles están constituidos por una tensión entre dos principios básicos: Límite e Ilimitado, ambos surgidos del Uno, que no es una remota e infinita abstracción metafísica, sino un poder que permanece continuamente presente en el horizonte de lo que es. Mientras el Límite corresponde a una disposición lógica precisa, lo Ilimitado es una corriente infinita que fluye a través del universo proveyendo vida y poder. ${ }^{7}$ La primera de las proposiciones expuesta en Elementos de Teología aporta una idea central: la totalidad de lo que es, en su multiplicidad, participa de la unidad ${ }^{8}$.

Paso a paso Proclo va deduciendo la existencia del Uno, y luego de todos los niveles, leyes y categorías del universo neoplatónico. Mientras en los Elementos procede por medio de deducciones puras y sin referencias históricas, en Teología Platónica ${ }^{9}$ toma el curso opuesto, en un trabajo de hermenéutica teológica de lo escrito por Platón acerca de los dioses de las teogonías órficas y caldeas. Otro grupo de obras de Proclo está constituido por los Comentarios, que para los neoplatónicos tardíos fue el género más importante de todos ${ }^{10}$.

El propósito de este trabajo es abordar en forma breve la continuidad descendente e íntimamente conectada que se va desplegando en el universo a partir del Uno-Bien, siguiendo en gran medida la lógica de la causalidad presente en Elementos de Teología, combinando la reflexión con ideas centrales presentes en otros textos.

\section{La identidad Uno-Bien y la causalidad}

El primer principio considerado por el autor es el Uno (que se identifica con el Bien) ya que toda multiplicidad es posterior al Uno, a la unidad: "

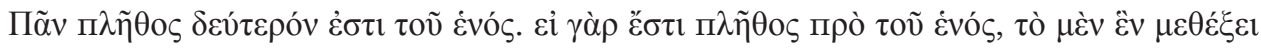

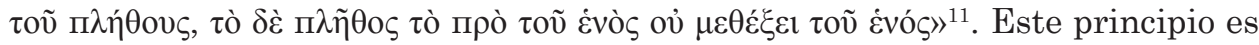
incausado; no puede decirse que el Primer Principio sea causa sui pues su ab-

[6] En efecto, metodológicamente esta obra está inspirada en Los Elementos de Euclides, ed. crítica de M. L. Puertas, Madrid: Gredos, 1996.

[7] Cfr. Radek Chlup, Proclus. An introduction, p. 48.

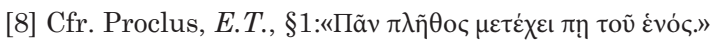

[9] Cfr. Proclus, Théologie Platonicienne, ed. H. D. Saffrey and L. G. Westerink, 6 vols, Paris: Société d'édition "Les belles lettres", 1968-97.

[10] Sobre el tema se puede consultar Ineke Sluiter, "Commentaries and the Didactic Tradition", Glenn W. Most (ed.), Commentaries - Kommentare (Aporemata , 4), Göttingen: Vandenhoeck \& Ruprecht, 1999, 173-205.

[11] Cfr. Proclo, E.T., §5.

THÉMATA. Revista de Filosofía, Nº 49 enero-junio (2014) pp.: 105-125

doi: 10.12795/themata.2014.i49.06 
soluta trascendencia -la consideración del Uno según el método de la dialéctica negativa ${ }^{12}$ - lo impide. Causalidad implica necesidad, y el Uno, en sí mismo no necesita nada para darse un principio, pues es intemporal y eterno.

La causalidad es una correlación transitiva en la cual sus términos son entidades sustanciales. Para los neoplatónicos se trata siempre de una relación transitiva, entre dos términos que se corresponden: el que produce y el que es producido. Pero esta categoría no envuelve una secuencia temporal, pues la relación no se da entre eventos, y por lo tanto no implica necesariamente una secuencia en el tiempo. Así, el principio de esta secuencia no tiene comienzo ni origen, así como tampoco lo tiene el universo, y esto es posible a raíz de la específica comprensión relacional de la causalidad que plantea el autor.

Toda causa permanece inmutable al constituir los seres subsiguientes. Por lo cual tampoco es acertado comprender la teoría procleana de la sucesión de causas y efectos como emanacionista. En efecto, en la ley que atraviesa todo el neoplatonismo y según la cual la causa da lugar a un efecto, la primera permanece sin disminución y sin alteración. Proclo agrega a esta noción de

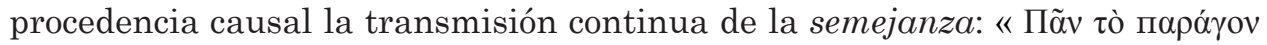

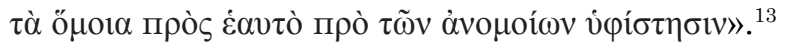

La semejanza en Proclo es la capacidad de mantener el carácter del origen en un menor grado. Esta semejanza inicialmente es una característica entre objetos, pero aquí posee un carácter necesario en la procesión de los seres, en la transmisión de lo mayor a lo menor, o de lo anterior a lo posterior. De esta manera, la semejanza requiere una derivación tal como se aduce en el parágrafo $18^{14}$, donde afirma que lo contenido en el efecto se encontraba ya en la causa. Asimismo, en el parágrafo 65 (E.T.) Proclo explica que todo lo que existe

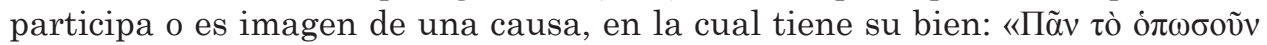

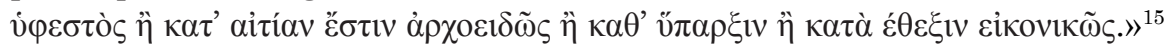

Una causa da lugar a ciertos efectos y no a otros. Tales efectos sólo reflejan parcialmente su principio productor, aunque es patente en ellos la semejanza que guardan con la causa. En Proclo la entidad producida por un agente causal es en sí misma capaz de producir algo fuera de sí, y si no es capaz de

[12] El método de la dialéctica negativa corre paralelo al de la dialéctica analógica, de la que ya se había mencionado más arriba en términos generales. El fundamento ontológico de la dialéctica analógica consiste en una semejanza entre el derivado y el Principio. La analogía es una tentativa de pensar el Principio, quien verdaderamente es Impensable e Inefable. Reparar en el Uno desde una perspectiva negativa significa adoptar el método más adecuado a la imposibilidad de comprensión de la esencia del Principio. Cfr.Werner Beierwaltes, Proclo. I fondamenti della sua metafisica, trad. de Nicoletta Scotti, Introd. de Giovanni Reale, Milano: Pubblicazioni della Università del Sacro Cuore, 1988, p. 371.

[13] Proclo, E.T., §28.

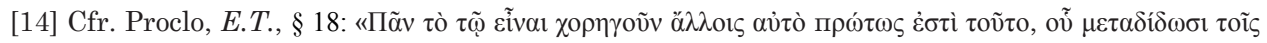

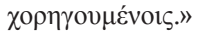

[15] Proclo, E.T., § 65.

THÉMATA. Revista de Filosofía, Nº 49 enero-junio (2014) pp.: 105-125 doi: 10.12795/themata.2014.i49.06 
ello, no es: esto se aplica a todos los niveles de la jerarquía de seres. En efecto, si no es, este mismo hecho determina su extrema inferioridad con respecto a su causa, es decir, se trata del último peldaño de la jerarquía. La materia es lo último en la cadena causal, y, como tal, es en cierta forma un no-ser, por lo cual no puede producir nada.

Lo máximo de esta escala conformada es el Supremo Bien. El texto

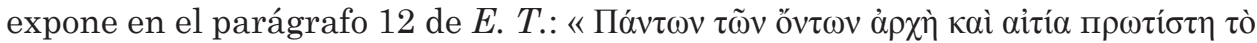

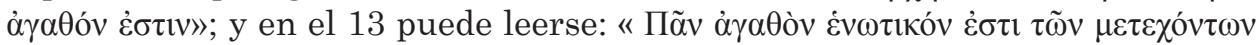

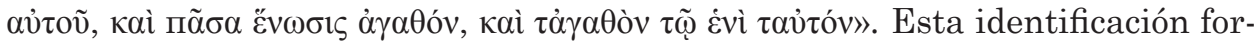
mal del Uno con el Bien deriva de Platón - de la cual también proviene la de Plotino-, según el cual, el Bien, por ser tal, por su propia naturaleza es pura comunicatividad. Tanto Plotino como Proclo no consideran la actividad creadora como aquello en que todo ha sido producido por libre elección. Para ambos, el que esta creación hubiera sido por libre elección hubiera significado que el Bien se puede concebir como aún no habiendo conferido algo, lo que implica que puede no haber sido totalmente Bien. Ellos entienden que los actos del UnoBien responden a una ley de su naturaleza. La ley de la Bondad sería la ley de la naturaleza de Dios ${ }^{16}$.

De manera que no se puede pensar -dentro de los límites de la filosofía procleana- que hubo un tiempo en que el Bien no había generado sus efectos; no es posible que no existiera el mundo con sus creaturas. No es pensable porque Proclo acentúa el Bien como la Primera Gran Causa. La causa es tal porque siempre es causa de sus efectos, pero no se debe entender la relación causa-efecto al modo de los pensadores modernos, quienes podrían concebir que dicha relación se identifica con la relación lógica de antecedente-consecuente.

\section{La realidad triádica en la jerarquía de seres y en los princi- pios de la comprensión de lo real}

En primer lugar se plantea la realidad en términos de jerarquía. Lo mismo que en Plotino, Proclo piensa en una procesión a partir del Uno, desde el cual surge una cadena de procesiones comenzando con los seres superiores hasta llegar a los inferiores. Se comprueba en esta concepción un tratamiento similar al del principio de la contigüidad ontológica ${ }^{17}$ y se pueden analizar sus

[16] Cfr. Taylor, "The Philosophy of Proclus", Proceedings of the Aristotelian Society, New Series, Vol. 18, London: Wiley-Blackwel, 1917-1918, p. 614.

[17] El principio de la contigüidad o continuidad ontológica será tratado más tarde en: Dionisio Areopagita, en Los Nombres Divinos, $1^{\circ}$ ed., trad. y notas por Pablo A. Cavallero, revisión y comentarios al texto por Graciela Ritacco, Buenos Aires, Losada, 2007, cap. VII. Este tema es desarrollado por Tomás de Aquino en varias de sus obras. Sobre esto, se puede consultar: Gabriela Caram, "Recepción y desarrollo del argumento de contigüidad ontológica en el Comentario a las Sentencias de Tomás de Aquino", Estudios Filosóficos, vol. LXII, N 181, Editorial San Esteban, 2013, pp.519-536.

THÉMATA. Revista de Filosofía, Nº 49 enero-junio (2014) pp.: 105-125

doi: 10.12795/themata.2014.i49.06 
particularidades, a saber: la realidad constituida en cuanto series encadenadas triádicamente y contiguas unas con otras.

En el contexto de un universo jeráquica y triádicamente organizado, Proclo sostiene la eternidad del mundo, declarando necesaria la conclusión de que el cosmos debe ser eterno, tomando el argumento del Timeo ${ }^{18}$, según el cual el demiurgo posee un hacer permanente, por el cual el cosmos siempre deviene, está en un continuo hacerse. La palabra "demiurgo", entraña el significado de "el hacedor", "el productor", "el artífice". A partir del argumento de Platón, el de Bizancio afirma que, a causa de que el demiurgo es causa eterna y divina, debe asimismo ser invariable: de lo que se sigue que si su naturaleza es ser "hacedor", siempre produce, y consecuentemente, el universo, siempre debe ser producido, no tiene comienzo ni fin. Por ser invariable, el demiurgo siempre desarrolla su obra en el mismo sentido, y por ello forja una única realidad articulada según estadíos de ser.

En este punto es necesario aclarar que Proclo considera al demiurgo como la primaria causa eficiente, responsable del orden y la estructura del universo. Es una figura divina cuyo rol es ser el "ordenador" del cosmos físico, cuyo status ontológico pertenece al reino del ser eterno. Es un intelecto divino, perteneciente al orden de los dioses intelectivos, anterior al alma ${ }^{19}$. Por lo tanto, vale aclarar, no es el Uno, sino una deidad ordenadora ${ }^{20}$.

En general, la figura triádica representa el ritmo en que se despliega el universo a partir del Principio. Proclo sostiene que la disposición triádica reproduce el orden de las relaciones por las cuales la más alta de las realidades está conectada con las realidades inferiores. Comienza la escala con la noción simplísima de Unidad o Uno, por ser el máximo y más pleno de todos los conceptos, y desciende en una serie de mentes, almas y cuerpos dispuestas

[18] Platón, Timeo, 27 d 6-28 a : «Qué es lo que es siempre y no deviene y qué, lo que deviene continuamente, pero nunca es? Uno puede ser comprendido por la inteligencia mediante el razonamiento, el ser siempre inmutable; el otro es opinable, por medio de la opinión unida a la percepción sensible no racional, nace y fenece, pero nunca es realmente. Además, todo lo que deviene, deviene necesariamente por alguna causa; es imposible, por tanto, que algo devenga sin una causa.»

[19] Cfr. Proclus, Commentary on Plato's Timaeus, Vol. II, Book 2: Proclus on the Causes of the Cosmos and its Creation, traducción, introducción y notas de David T. Runia y Michael Share, Cambridge: Cambridge University Press, 2008, p. 23: «Plato describes him as a nous at 39e7 (cf. $224.7,323.24$ ), as an eternally existent god at $34 \mathrm{a} 8$ (cf. 230.2), and he is to be identified with the 'Royal intellect' in Philebus 30d2 (cf. 224.1, 315.16, 406.29).»

[20] Cfr. Proclus, Commentary on Plato's Timaeus, p. 23: «(...)the Demiurge is an Intellect situated in the realm of eternal Being. More precisely he marks the border of the intellective gods.69 The transcendent realm of Being, situated at the level under that of the first Principle and connected to it by means of the henads, consists of three levels of gods, a triad of intelligible gods (to which the Paradigm belongs), a triad of intelligible-intellective gods which are the cause of life, and thirdly seven intellective gods, of which the Demiurge is the lowest of the first triad. This god only is called 'Maker and Father' by Plato (311.26).»

THÉMATA. Revista de Filosofía, Nº49 enero-junio (2014) pp.: 105-125 doi: 10.12795/themata.2014.i49.06 
en tríadas hasta los seres más imperfectos ${ }^{21}$. El método por el que las tríadas se van sucediendo es inmediatamente inteligible, y su primer principio es una Absoluta y Trascendente Deidad que es la fuente de todos los existentes, sus caracteres y sus relaciones entre ellos.

En síntesis, la tríada implica una unidad subsistente en la multiplicidad y una unidad subsistente en la diferencia. Por ello es unidad dinámica que se contrapone a la identidad lógico-estática o tautológica. El carácter de dinamicidad lo da el orden según una subordinación en serie.

\section{Las tríadas}

Proclo estima que del Uno procede todo lo que es, ordenado en tríadas descendentes y jerarquizadas, donde el primer miembro de cada una de ellas es no-participado ( $\dot{\alpha} \mu \varepsilon \dot{\varepsilon} \theta \varepsilon \kappa \tau \varsigma \varsigma)$. Se trata de principios genéricos, a los que denomina mónadas, de los cuales derivan los elementos participados de cada tríada. El autor concibe el cosmos como un todo en el que cada miembro de la sucesión da lugar a otro. Los primeros términos generan una serie de entes del mismo tipo que el primero. Es así como al nivel del Intelecto, por ejemplo, surge una serie de intelectos más o menos elevados, y similarmente, en un nivel inferior, una serie de almas y una serie de cuerpos con mayor o menor valor y dignidad. La serie que sigue al miembro principal recibe predicación, es decir, son inteligencias o almas participadas. Así, en el caso de los intelectos, el primer miembro de la serie es el Intelecto, y los demás son intelectos de los seres que tienen intelecto.

Esta especulación es aplicable al Bien o Uno, no menos que al Intelecto o al Alma. Si el Intelecto da lugar no sólo al Alma, sino a una pluralidad de intelectos, el Supremo Uno o Bien debe ser pensado como dando lugar a una serie de "unos", a los cuales Proclo llama "hénades", que son divinidades, o unidades divinas, que pertenecen al reino del Uno, como lo primero derivado. Como el Bien es Dios, en este sistema las hénades son divinidades en plural ${ }^{22}$, relacionados con Dios así como los intelectos se relacionan con la entidad que llama Intelecto o Inteligencia. Dios aparece como la fuente de cuatro órdenes: hénades, intelectos, almas y cuerpos. Ellos forman una jerarquía de "imágenes" a la manera de reflejos del Uno. Y cada orden forma una jerarquía de imágenes o reflejos de su miembro inicial propio. La tríada del cosmos surgida del Uno se constituye sintéticamente así: del Uno y del reino de los "unos" o hénades, surgen la Inteligencia, el Alma, y el Cuerpo, en cuanto mónadas o realidades

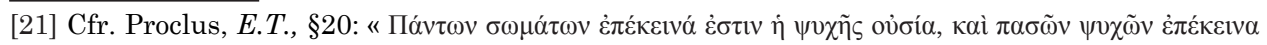

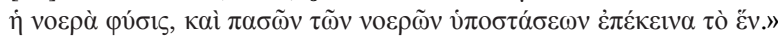

[22] Las hénades son dimensiones universales de la actividad de Dios. Cfr. Edward P. Butler, The Metaphysics of Polytheism in Proclus, United States: UMI, 2003, p.8.

THÉMATA. Revista de Filosofía, Nº 49 enero-junio (2014) pp.: 105-125

doi: 10.12795/themata.2014.i49.06 
inteligibles que son paradigmas de los entes ${ }^{23}$. Estas tres unidades monádicas o formas paradigmáticas dan lugar a una serie de individuos: intelectos, almas y cuerpos particulares.

El sistema completo es una ordenada serie de ordenadas series, donde cada ser tiende a su bien, lo cual constituye la ley de su ser. Proclo afirma que es una consecuencia de la identidad del Uno con el Bien que todas las cosas tiendan a él en un movimiento de retorno, en busca de su propia simplificación. De este modo las cosas tienden a una afirmación de unidad e individualidad, en la medida en que su naturaleza lo permite: en este proceso de reversión, la individualidad no se rompe, ni los cuerpos dejan de ser tales, así como las almas tampoco.

Vale aclarar que el retorno de los seres al Uno no significa que aquellos llegan a ser Dios, sino que en su contemplación de sí mismos, aprenden a conocer a Dios, en la medida en que es comprensible a algunas de sus creaturas. No se trata de un Absoluto en el cual algún tipo de individualidad infinita sea transformada, sino que toda cosa es lo que es y no otra cosa, sin cesar de ser lo que es. ${ }^{24}$

\section{1. La procesión de las tríadas inteligibles}

En el sistema procleano la organización de la realidad en tríadas tiene variados alcances. Por un lado, encontramos la realidad ordenada como se vio anteriormente en tres niveles encabezados por hénades procedentes del Uno, constituyendo una cadena jerárquicamente dispuesta de seres. Paralelamente, Proclo desarrolla un sistema hermenéutico de tríadas inteligibles, a la manera de explicaciones también triádicas y concatenadas entre sí, concernientes a la comprensión de la dinamicidad estructural del sistema de lo real. El pensamiento explica, en este ordenamiento, la forma en que ha procedido la realidad desde el Uno, a su vez desde distintas perspectivas de interpretación. Se contempla la realidad según sus aspectos, que resultan inmediatamente inteligibles en la explicación secuenciada de Proclo. Este método de análisis no constituye una mera funcionalidad formal, sino que aparece como expresión de la estructura de lo real: la estructura triádica del pensamiento se vincula esencialmente con la disposición metafísica de lo real, y sus elementos se van sucediendo gradualmente así como ocurre en la dimensión de la existencia.

El fundamento unitivo de las tríadas es la posibilidad de la comuni$\operatorname{dad}^{25}$ (Kotvovía) de las cosas en cuanto modalidad en la cual el espíritu (voṽc) re-

[23] Para la profundización en el origen y distinción entre hénadas y mónadas ver Edward P. Butler, The Metaphysics of Polytheism in Proclus, cap. 4, pp 151-185.

[24] Cfr. Taylor, “The Philosophy of Proclus”, p. 633.

[25] Cfr. Werner Beierwaltes, Proclo. I fondamenti della sua metafisica, p.78.

THÉMATA. Revista de Filosofía, Nº 49 enero-junio (2014) pp.: 105-125 doi: 10.12795/themata.2014.i49.06 
duce los tres elementos de la tríada en una unidad; es decir que el pensamiento se despliega como su principio ontológico, y a la vez hermenéutico unitivo. En verdad se da una conexión no externa o meramente causal, sino una unidad subsistente de la triadicidad consigo misma, en que la intencionalidad del pensamiento permanece preservada.

El ordenamiento de las tríadas hermenéuticas o pertenecientes al reino

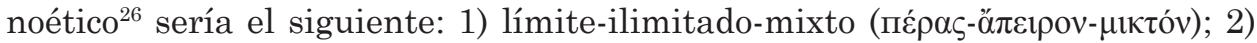

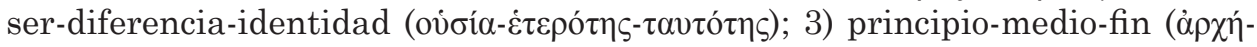

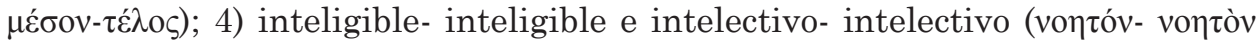

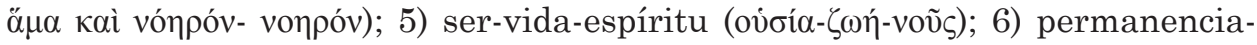

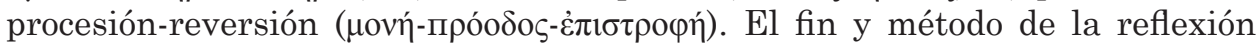
en torno a este orden es mostrar, primeramente, cómo la esencia de la tríada constituye la estructura básica del sistema del ente, y en la estructura triádica del ente encuentra su fundamento el desarrollo triádico del pensamiento.

\section{Límite-ilimitado-mixto ( $\pi \varepsilon ́ \rho \alpha \varsigma-\alpha ̈ \pi \varepsilon ı \rho o v-\mu 1 \kappa \tau o ́ v)$}

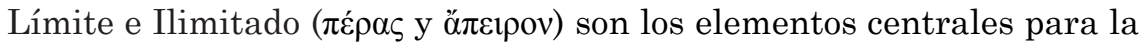
explicación de la producción de la multiplicidad a partir de la Primera Causa. Esta parece ser la primera preocupación de Proclo, quien no concibe "saltos" entre una u otra fase de la realidad. En primer lugar, el Uno sólo puede producir unidad, y esta unidad se cristaliza en el principio del Límite. Éste define los límites del ser,

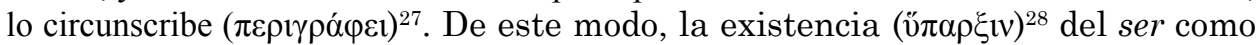
una entidad separada es el resultado del primer Límite. Esta acción limitante, consiste en la unificación que es requerida para la existencia de un ser, el cual, por sí mismo, no es capaz de constituirse en una unidad separada.

Así como el Límite, el poder generativo de lo ilimitado va constituyendo una serie que tiene miembros en cada nivel de realidad. Cada ser es productivo en un sentido u otro, en cuya serie el gobierno es ejercido por el principio de la

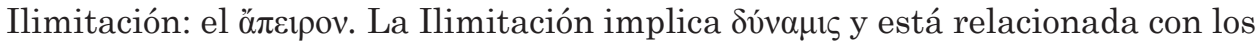
grados más altos de la realidad, aunque la jerarquía posee en sus niveles, diferentes manifestaciones de ésta, así como el öлєıрov se desarrolla en diferentes grados.

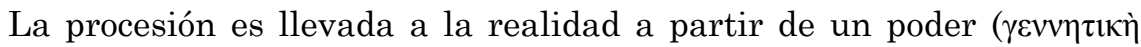

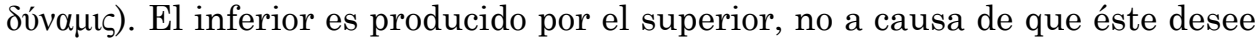

[26] Cfr. Sarah Klitenic Wear y John Dillon, Dionysius the Areopagite and the Neoplatonist Tradition, England: Ashgate, 2007, p. 25.

[27] Cfr. Proclo, In Platonis Cratylum commentaria, ed. G. Pascuali, Lipsiae, 1908, 42, 13, 21-22 y $24-26$.

[28] Cfr. Proclus, In Platonis Timaeum Commentaria, Ed. E. Diehl, 3 vols., Leipzig: Teubneri,

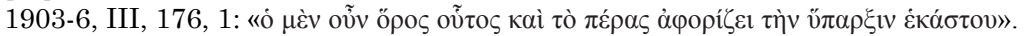

THÉMATA. Revista de Filosofía, Nº49 enero-junio (2014) pp.: 105-125

doi: 10.12795/themata.2014.i49.06 


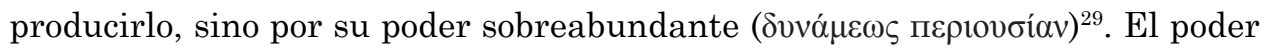
del superior resulta incomprensible para el inferior, en el cual la multiplicidad

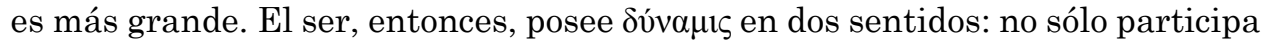

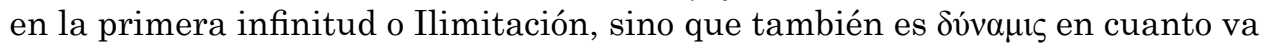
produciendo el nivel subsiguiente. La sucesión de los diferentes niveles es explicada como la actualización de aquello que estaba potencialmente en el grado previo $^{30}$; aquí se puede corroborar la continuidad sin hiato intermedio entre los niveles de la realidad.

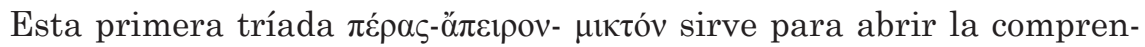
sión en torno al funcionamiento de los tres elementos que conforman la primera realidad intermediaria que surge del Uno. Como se ha visto, a partir de dos elementos que van surgiendo uno del otro se genera una relación, sintetizada en el tercer término. En el esquema de Límite, Ilimitado y Mezcla ( $\mu 1 \kappa \tau o ́ v)$, el tercer término es producido, y propiamente, sólo realiza la combinación de los dos anteriores. Pero en cierto sentido, la unidad que caracteriza al tercer elemento parece ser débil para excluir la dualidad. ¿Pero cómo sería posible una dualidad en el nivel de realidad que está justo por debajo del Uno y su reino henádico-es decir, la primera tríada-? ¿No debería ser aquel nivel que tuviera la mayor unidad?

Proclo comprende que por encima de las distinciones es posible salvaguardar la unidad. Su filosofía, en sí, constituye, a pesar de las diferenciaciones continuas, un esfuerzo de unificación permanente de todo el espectro de lo

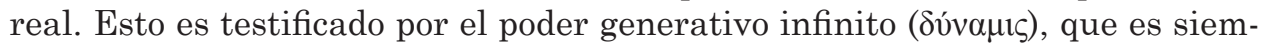
pre inherente al límite. La ilimitación nunca existe apartada del límite; Límite, Ilimitación y su combinación reaparecen en todos los niveles de la realidad.

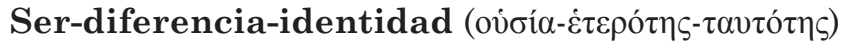

De la segunda tríada, el ente en la dimensión de la ovoía es una unidad de contraposiciones, porque en ella se compenetran Límite e Ilimitación, Identidad y Diferencia, ser y no ser. Así, la realidad total está gobernada por la dualidad, desde lo más alto hasta lo inferior. Al llegar al final de la sucesión, la materia concluye la manifestación más pura de lo Ilimitado; es el último nivel, pero es cabalmente miembro de la serie, por lo cual se puede colegir que ha sido producida por el Uno Bien, como su primera e inefable causa. ${ }^{31}$

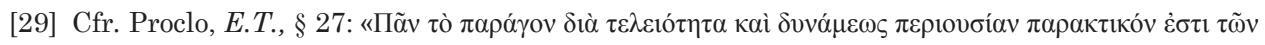

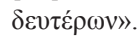

[30] Cfr. S. E. Gersh, "From Iamblicus to Eriugena. An investigation of the Prehistory and Evolution of the Pseudo Dionysian Tradition", en Studien zur Problemgeschichte der antiken und mittelalterlichen Philosophie, 8, Leiden: Brill, 1978, pp. 32-33.

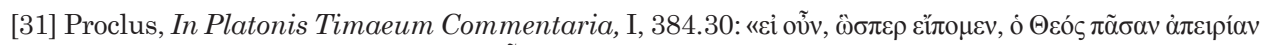

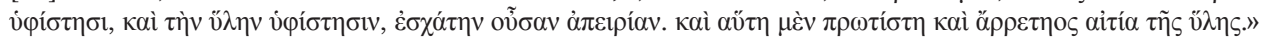

THÉMATA. Revista de Filosofía, N 49 enero-junio (2014) pp.: 105-125

doi: 10.12795/themata.2014.i49.06 
De este modo, también puede concluirse que la ausencia de determinación no puede estar relacionada absolutamente con el mal. Por el contrario, se ha mostrado que se origina del Bien, por lo cual es imposible asociar la materia, y por lo tanto, los cuerpos y la ilimitación, con el mal. Toda la naturaleza material debe ser referida de continuo a la causa, porque hasta ella ha llegado

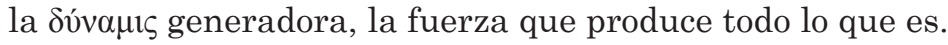

La indeterminación en cuanto potencia generatriz es principio de la procesión, constituyéndose en el fundamento primario por el cual la multiplicidad de los entes es externa al Uno y la potencialidad que preexiste en el límite se comunica al ente, mientras al mismo tiempo el límite actúa articulando la multiplicidad.

Proclo indica la unidad de Límite e ilimitado como clave para entender el principio de la constitución del ser. Ser, identidad y diferencia, penetran también la totalidad de la realidad. En cada cosa existente, identidad y diversidad resultan unidas en virtud del ser; juntas determinan lo indefinido a formar tal o cual cosa. Límite y diferencia, a su vez, ponen en evidencia el no ser de la identidad en relación a lo distinto.

Como la identidad y la diferencia no son opuestos contradictorios, del mismo modo, el no ser no significa nulidad pura, sino un no ser que radica en los varios tipos de relaciones que acaecen entre las cosas. La diferencia es principio y causa de separación y división. El poder de la diferencia pone la distinción ya que hace que cada ser sea él mismo y no otro en virtud de la negatividad del límite. La identidad, en cambio, reconduce la alteridad en la unidad ya diferenciada, y funda su esencia, en un sentido positivo, definiéndola, y en un sentido negativo, separándola. ${ }^{32}$

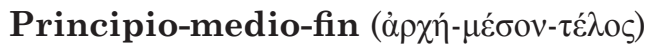

La tríada principio-medio-fin se encuentra en el fundamento de la esencia de la totalidad que en todas las cosas se manifiesta y las presenta en cuanto definidas y unitarias. Pero propiamente esta tríada explica al Uno en su ser principio supraexistente, por el cual cada realidad tiene su propio inicio, el propio punto medio o potencia que lo mantiene en el ser -porque en ella está centrado y radicado-, y el propio fin al cual nuevamente debe retornar para encontrar su cumplimiento. El Uno no tiene en sí inicio, medio ni fin, porque implicaría una relacionalidad consigo mismo, una autorreflexión del Uno, en la cual, permaneciendo en sí mismo saldría de sí por automediación, para retornar a sí nuevamente. En cambio, el Uno está fuera de toda determinación categorial, relacional o temporal; pero es el origen de la tríada presente en las cosas, y en ellas está como su principio, medio y fin. Como Platón dice en Leyes $^{33}$, Dios tiene en sus manos el principio, el medio y el fin de todo lo que existe.

[32] Cfr. Werner Beierwaltes, Proclo. I fondamenti della sua metafisica, p.111.

[33] Cfr. Platón, Diálogos. VIII. Leyes (Libros I-VI), Madrid: Gredos, 1999, 715 y ss.

THÉMATA. Revista de Filosofía, No 49 enero-junio (2014) pp.: 105-125

doi: 10.12795/themata.2014.i49.06 


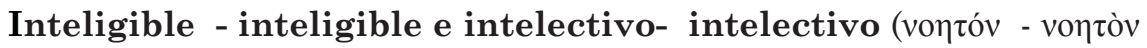

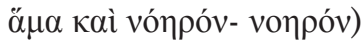

Con respecto a la cuarta tríada inteligible- inteligible e intelectivo -intelectivo, primeramente debe comprenderse que la diferenciación ontológica y la articulación jerárquica en la esfera del espíritu (voṽs) tuvo su inicio en Jámblico,

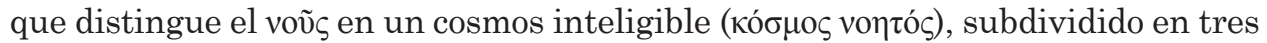

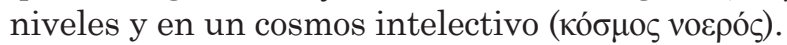

El término vontóv explica la inteligibilidad de un ente, su inteligible determinación; el ente en cuanto real se funda en el voũ pensante, esto es, en cuanto es pensado. El término vocpós indica, en cambio, el inteligir, el acto del intelecto o del espíritu, que no es simplemente pensamiento, sino el pensante; el pensamiento se cumple siempre en el espíritu. Indica la dirección de sentido o de intencionalidad del voṽ , el cual no puede ser comprendido como un vacío totalmente privado de contenido, de determinaciones, de diferencias y de relaciones ${ }^{34}$, sino que es una unidad relacional de todo lo pensado en cuanto plenitud de la idea, y siempre unido a un inteligible (voๆtóv) inteligiblemente determinado ${ }^{35}$. Así, el espíritu no es otra cosa que ser, y en esta identidad acontece una tríada (pensado -pensado junto al pensante -pensante): al mismo tiempo espíritu y ser, pensante y pensado son idénticos

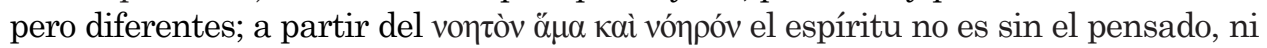
el pensado existe separado del espíritu, y no queda nada fuera de esta relación como unidad intencional. Esta tríada se completa en su continuidad con la siguiente.

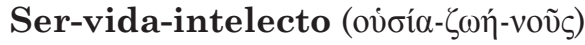

$\mathrm{El}$ orden inteligible toma su forma fundamentalmente de esta tríada ${ }^{36}$. En la interpretación del voṽ s por medio de la tríada ser-vida-pensamiento o espíritu, se ve que este pensamiento, en cuanto que es, es ser; lo inteligible es el ser. En virtud de la intencionalidad de ambos, la identidad de ser y pensamiento relacional: el ser es siempre ser del pensamiento, y éste es siempre pensamiento del ser. El espíritu, en el cual esta unidad se realiza, es el ser que piensa, mientras que el ser, en su ser pensado, confiere al voṽ s pensamiento y vida. El parágrafo 103 de Elementos de Teología refiere que todas las cosas están ordenadas de tal modo que en el ser hay vida e inteligencia; en la vida hay ser e inteligencia; en la Inteligencia hay ser y vida; pero cada una de ellas existe en distintos niveles: en un nivel intelectual, en otro de modo vital y en el tercero existencialmente. ${ }^{37}$

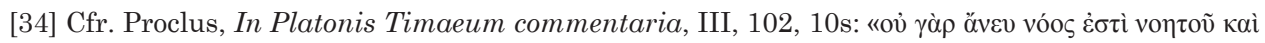

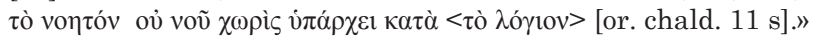

[35] Cfr. Werner Beierwaltes, Proclo. I fondamenti della sua metafisica, p. 135.

[36] Cfr. Edward P. Butler, The Metaphysics of Polytheism in Proclus, pp. 10-11.

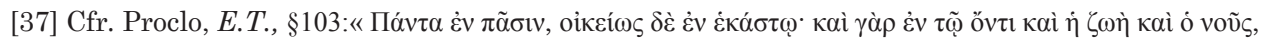

THÉMATA. Revista de Filosofía, Nº49 enero-junio (2014) pp.: 105-125

doi: 10.12795/themata.2014.i49.06 
El ser es un elemento dinámico para la vida y el pensamiento: éstos pueden desplegarse en el ser y por medio del ser, como vida existencial y pensamiento existencial. El pensamiento, como tercer elemento, está en relación con el ser y la vida en virtud de su participación en ellos. En efecto, el ser puede ser pensado sin la vida y sin el pensamiento; la vida puede ser pensada sin el pensamiento, pero éste no puede ser concebido sin el ser y sin la vida. Sin el acto de la vida que opera la mediación entre los dos elementos, el espíritu no tendría ninguna conciencia del propio ser y de la propia esencia. El ser, que es mediado con el pensamiento mediante la vida, es la vida del pensamiento, mientras el pensamiento es la vida del ser, y con mayor razón, el pensamiento es el ser de la vida ${ }^{38}$. Ella, en cuanto pensante y existente, funda el acto de identidad que ocurre entre el ser y el espíritu, y deviene mediadora de la triadicidad del voũc.

\section{Permanencia- procesión-reversión (}

El desenvolvimiento de esta tríada, es fundamental para el pensamiento procleano y para los pensadores que reflejaron posteriormente parte de su filosofía. Refleja con claridad una impronta basal de pensamiento que aparecerá hasta el neoplatonismo de Tomás de Aquino y el posterior.

La esencia y la acción de la tríada permanencia-procesión-reversión no halla su ubicación apropiada junto a las otras tríadas, sino que es aquella que se encuentra ínsita en las demás, y constituye su fundamento dinámico. Es decir que se revela como fundamento del dinamismo de la unidad del cosmos, como causalidad inteligible, como despliegue desde la Idea comprendida en el voũ del demiurgo en la multiplicidad sensible y como cíclico retorno del mundo a su propio fundamento inteligible.

Se constituye especialmente, además, en un principio estructural del espíritu que procede transversalmente a través de los momentos que lo componen. Funda el movimiento del pensamiento como reflexión en relación con el propio pensamiento, y por lo tanto, produce un retorno a sí mismo. Por la dimensión del espíritu, la permanencia significa el ser en sí, el permanecer en sí del espíritu, que es por esencia autosubsistente ${ }^{39}$, lo cual no significa que el espíritu sea el fundamento absoluto de sí mismo, pero en cuanto hipóstasis fundada en sí misma no necesita de ninguna otra hipóstasis para la realización de la propia esencia. Su permanecer en sí no guarda relación con la dimensión temporal ni espacial. Ni tampoco la procesión del espíritu se desarrolla en una superación de su propia dimensión; no implica movimiento discursivo, sino que

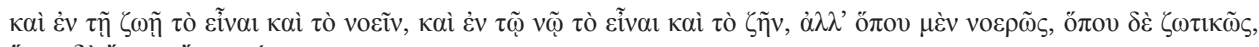

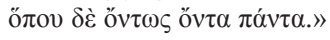

[38] Cfr. Werner Beierwaltes, Proclo. I fondamenti della sua metafisica, p. 141.

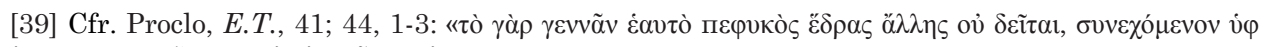

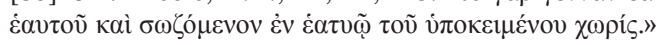

THÉMATA. Revista de Filosofía, No 49 enero-junio (2014) pp.: 105-125

doi: 10.12795/themata.2014.i49.06 
abraza todo -coincide con lo pensado-, porque la inteligencia tiene intelección simultánea de todas las $\operatorname{cosas}^{40}$. Su intelección abarca todas las cosas perpetuamente, y en todas las inteligencias, pero en cada una de ellas delimita todos sus objetos por un carácter particular. Así, el autocumplimiento de su esencia es un dinamismo que permanece en sí diferenciándose, pues todo lo que es autohipostático es capaz de reversión sobre sí mismo. ${ }^{41}$

El movimiento de esta tríada tiene lugar a partir de un nexo de causalidad, en el cual todo lo que procede de cualquier principio revierte, respecto de su ser, sobre aquello de que procede ${ }^{42}$. Esta reversión se produce con motivo de que cada cosa desea su bien y cada una de ellas lo alcanza por mediación de su causa próxima; es decir que el objeto primario de su apetito es aquello sobre lo que revierte. Esta última tríada hace patente el sistema causal procleano. Una vez comprendidas las tríadas noéticas que secuencialmente contempla el pensador, es necesario considerar el ordenamiento de lo real.

\section{2. La procesión de lo real a partir del Uno}

\section{Las hénades}

La identificación de las hénades con ciertas divinidades puede ser atribuida al maestro de Proclo, Siriano ${ }^{43}$. Como entidades metafísicas, se comprende que son divinas "unidades" o "unicidades" que poseen el aspecto de una unidad trascendente.

El pensador de Bizancio se vale de esta noción para indicar la existencia de entidades intermedias, para explicar la continuidad de la procesión de seres desde el Uno hasta la realidad múltiple. Las hénades están para abrir un puente en el abismo que Plotino pensó entre el Uno y la realidad. Son el primer derivado del Límite y la Ilimitación, los cuales cumplen la función de principio y causa con respecto a la totalidad de lo real. Son principios de los cuales participa escalonadamente, según sus posibilidades, el reino del ser; son unidad múltiple, o multiplicidad unitaria, originaria y primera, luego del Uno absoluto.

Las hénades o divinidades proceden del Uno y dan lugar a los primeros términos de las tríadas existentes. Trascienden los miembros de cada tríada como realidades separadas de las que participan los componentes subsiguientes. Los principios divinos, de este modo son más comprehensivos y más potentes que lo que procede de ellos, y es a causa de la falta de idoneidad de los

[40] Cfr. Werner Beierwaltes, Proclo. I fondamenti della sua metafisica, p. 163.

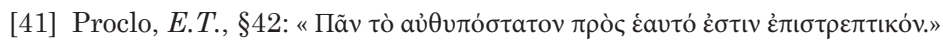

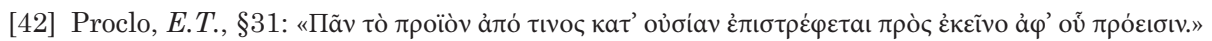

[43] Siriano fue sucesor de Plutarco de Atenas y uno de los representantes del neoplatonismo plotiniano de la Escuela de Atenas.

THÉMATA. Revista de Filosofía, N 49 enero-junio (2014) pp.: 105-125

doi: 10.12795/themata.2014.i49.06 
demás miembros de cada serie lo que da lugar a la insuficiencia de luz divina que llega a ellos. Cuando esta luz se debilita, se debe a la impotencia del participante, y parece asumir la dirección otro principio o hénade. ${ }^{44}$

Estas deidades ejercen su providencia con las existencias secundarias y a su vez las trascienden, sin perder su unidad, y sin que su separada unidad anule su trascendencia, pues su carácter distintivo es la irradiación de la bondad. Suministran el bien sin ninguna concesión calculada, pero los participantes inmediatamente inferiores reciben según su mérito y dignidad ${ }^{45}$. En su calidad de trascendentes son inefables e incognoscibles por cualquier ser secundario a causa de su unidad supraexistencial, pero pueden ser aprehendidas y conocidas de algún modo a partir de los existentes que participan en ellas. Por esto, solamente el Uno es absolutamente incognoscible, por ser no participado. ${ }^{46}$ Una afirmación claramente neoplatónica desde el punto de vista gnoseológico es que, en términos generales, el nivel más alto es sólo cognoscible por medio de sus efectos en lo inferior. ${ }^{47}$

Así, la hénade es co-operante con el Uno en la producción del existente real que participa de ella. De tal modo que todas las capacidades de los dioses proceden a través de los intermediarios y descienden hasta los últimos existentes de las regiones terrestres. La hénade es divinidad inmediata, la inteligencia es divina en grado máximo, el alma es divina; el cuerpo es deisimilar. El carácter divino sucesivamente descendente de los grados de ser procede de la

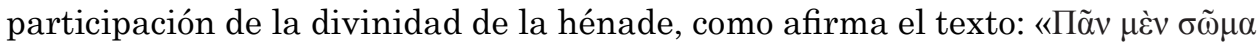

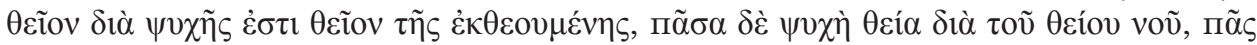

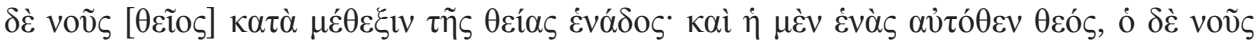

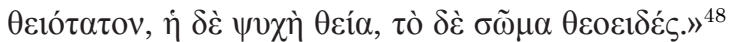

Esta concatenación va llevando desde el Uno lo máximo de plenitud que desciende en cada ser a su modo por la mediación primera de las hénades, de las cuales participan los demás términos.

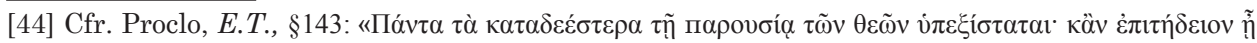

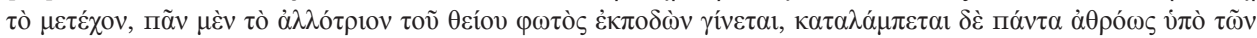
$\theta \varepsilon \tilde{\omega} v . »$

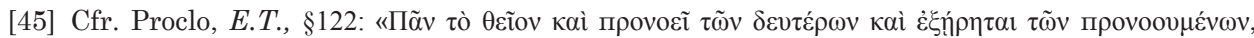

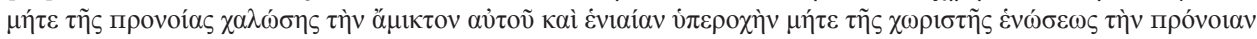
$\dot{\alpha} \varphi \alpha v i \zeta o v ́ \sigma \eta \zeta . »$

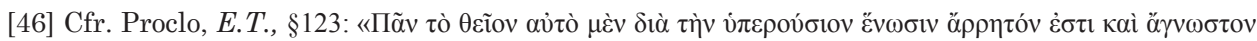

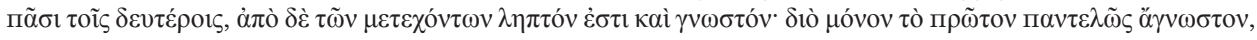

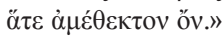

[47] Cfr. Taylor, “The Philosophy of Proclus”, p. 632.

[48] Cfr. Proclo, E.T., §129.

THÉMATA. Revista de Filosofía, Nº 49 enero-junio (2014) pp.: 105-125

doi: 10.12795/themata.2014.i49.06 


\section{Intelecto, Alma y Cuerpo}

La Inteligencia es una existencia indivisible porque es incorpórea, lo cual se demuestra porque puede revertir hacia sí misma, mientras que los cuerpos son incapaces de reversión; produce sus consecuentes mediante el acto de su intelección, siendo su actividad creadora el pensar, puesto que pensamiento es creación. ${ }^{49}$

Proclo habla de la Inteligencia Primaria, participada por las demás inteligencias. En el caso del Alma, es participada por las almas particulares; se entiende que son sustancias incorpóreas, independientes del cuerpo, pero dependientes de la inteligencia. "Toda alma tiene su origen inmediato en una inteligencia", de manera que toda alma participada tiene una existencia eterna, a raíz de su origen, pero una actividad propiamente temporal.

En cuanto a su lugar propio en la jerarquía, la actividad del alma consiste en comunicar al nivel inmediatamente inferior su proprium, que es la vida. Vivifica los cuerpos infundiendo sobre ellos su propia virtud, otorga la vida para dotar de un especial tipo de unidad y organización características de los organismos. No todos los cuerpos son capaces de recibir este tipo de unidad y estructura, sino sólo algunos, los que poseen materia orgánica. En la materia inorgánica, el alma no puede ya ejercer ninguna influencia, y este tipo de realidad ocupa el último lugar en el sistema de los existentes. Solamente es capaz de revertir sobre sí mismo aquello que es incorpóreo, por lo cual los cuerpos no pueden hacerlo, y allí encuentra su final la gran cadena de procesiones del universo. ${ }^{50}$

Como primeros términos de serie, el Intelecto y el Alma, generan una serie de entes del mismo tipo que ellos mismos, con lo cual, al nivel de la Inteligencia, se genera una serie de inteligencias menos exaltadas, $y$, similarmente, en el horizonte del Alma, una sucesión de almas y, subsiguientemente, en el ámbito del cuerpo, una serie de cuerpos de mayor o menor valor y dignidad.

Proclo integra completamente la materia en su ontología, y evita hacer una excepción con respecto a la generación de ella a partir de las leyes de la causalidad. Por lo tanto, la materia deriva de la existencia del Uno, es producida por Él, intermediado por otros seres. Además, comprende que esta generación no puede ser comprendida como producida en el tiempo, sino como una dependencia de su causa, pues una producción divina debe ser eterna y sin movimiento. ${ }^{51}$

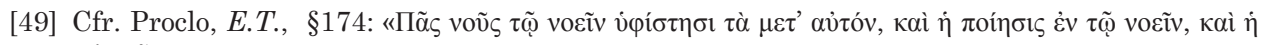

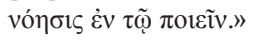

[50] Cfr. Radek Chlup, Proclus, p. 74.

[51] Cfr. De Haas, “John Philoponus' New Definition of Prime Matter. Aspects of its Background in Neoplatonism and the Ancient Commentary Tradition", Philosophia Antiqua, 69, New York -Köln, 1997, pp.7-8. 
En De malorum subsistencia ${ }^{52}$ (capítulos 11-38) Proclo explicita su postura con respecto a la materia, y fundamenta el rechazo a la consideración plotiniana de la materia como causa del mal en el alma. Refuta la idea de que la materia sea el principio de todos los males, y rebate la razón de Plotino de la materia como completa privación. Privación es contraria a la forma, y por lo tanto, su característica es no ser. Está en las antípodas de ser un substrato que desea una forma, y sólo accidentalmente no es. Así, el último rango de ser, el reino de lo material, deriva su poder del Bien, y no debe ser considerado como privación o como un mal, sino como un subcontrario del Bien, esto es, no como su contrario ${ }^{53}$. Así lo afirma en los capítulos 52 y 53 de la obra citada. El mal absoluto es incapaz de recibir una forma; por el contrario, la materia es un substrato sin cualidad ni forma, pero que las precisa. La materia no es buena ni es mala, sino necesaria. ${ }^{54}$ Se debe recordar que el Uno no sólo produce lo que es limitado, sino también lo ilimitado, cuyo último estadío es la materia.

\section{Conclusiones}

A lo largo de este desarrollo, la jerarquía planteada en las bases de este pensamiento, significa tanto la organización del universo, una interpretación de la organización del universo, como también un verdadero proceso. Como proceso, implica actividad; la actividad de la jerarquía es el acto de la creación del Uno, y un deseo de todo lo creado de retornar a la Unidad suprema. La tríada es el modo según el cual se articula el despliegue del universo a partir del mismo Principio en eslabones sucesivos.

Uno de los puntos principales del pensamiento neoplatónico es la conexión que guardan los diferentes niveles a través de una estructura gradualmente dispuesta, en la cual, el punto inferior de cada nivel de ser, es el más alto de los puntos del nivel que le sigue, resguardando la consideración de la unidad y trascendencia del Uno, y a su vez, colocando como su siguiente inmediato a la multiplicidad y a la creación. Muy particular del pensador de Bizancio es la búsqueda de disminución de toda distancia entre cada nivel de ser, y a su vez, en cada nivel del ámbito noético. Como se dijo, la estructura triádica del pensamiento (la interpretación de las tríadas inteligibles) encuentra su correspondencia en la ordenación triádica de la realidad.

Entre los paganos, solamente Proclo sostuvo la estructura triádica, que

[52] Proclo, De malorum subsistentia (cit. DMS), ed. H. Boese, Berlín: Berolini, 1960.

[53] Cfr. Phillips, John, Order From Disorder. Proclus' Doctrine of Evil and its Roots in Ancient Platonism, Edited by Robert M. Berchman and John F. Finamore, Boston: Brill, 2007, p. 70: «(...) in Theaetetus he employs the term "sub-contrary" to describe the relationship of evil to the Good as one of not total contrariety. Evil is not wholly so, which is to say that its opposition to the Good is not absolute, insofar as it has no power or activity of its own».

[54] Cfr. DMS, pp. 36-37.

THÉMATA. Revista de Filosofía, Nº49 enero-junio (2014) pp.: 105-125

doi: 10.12795/themata.2014.i49.06 
encuentra en Plotino sus primeras raíces, a la que puede comprenderse como una unidad que se desarrolla triádicamente o tríada unitaria. La multiplicación progresiva de dicha estructura a partir de los primeros principios es uno de los rasgos más notables de su filosofía. Este sistema constituye un proceso gradual y continuo, en orden a explicar la naturaleza de todas las cosas, y establecer un procedimiento en que la distancia entre los momentos sea lo más ínfima posible. En el universo procleano, la tríada fundamental Ser-Vida-Intelecto proviene desde el Uno, mediada por el Límite y la Ilimitación. Tanto para Siriano como para Proclo dicha tríada es una tri-unidad (trinidad) que aparece en el comienzo de lo que sería la segunda hipóstasis, de manera que después del reino del Uno, existen el Ser, la Vida y el Intelecto -tríada noética-, manifiestos en las hénades seguidas de intelectos y almas, hasta llegar al reino de la pura materia.

Es importante también observar que la tríada procesión-permanencia-reversión constituye un elemento visiblemente neoplatónico, que favorece la concepción del principio de la continuidad de los seres, posteriormente formulado por Dionisio Areopagita. Dentro del sistema, cada término procesivo singular revierte sobre su propio principio inicial, de quien procedió. Esta reversión del fin sobre el comienzo hace que todo el orden sea uno y determinado, convergente sobre sí mismo, manifiestando la unidad en la multiplicidad ${ }^{55}$. Se revela aquí un claro antecedente del argumento de contigüidad ontológica, expresado cabalmente en el parágrafo 147 de E.T. En él se muestra la continuidad a lo largo de las procesiones divinas; cada orden está ligado a otro por los términos medios adecuados. Los términos más elevados de la segunda serie están necesariamente unidos con los términos limítrofes del primero. La conexión específica de cada rango de ser se verifica por medio de la semejanza; por consiguiente, habrá semejanza entre los principios iniciales del orden inferior y los últimos miembros del más elevado, ${ }^{56}$ en el punto de unión entre lo inferior de lo superior y lo superior de lo inferior. Se comprueba una vez más el interés del pensador por mantener un encadenamiento sin fisuras entre los componentes de la realidad.

Además de la contigüidad ontológica, es posible descubrir una continuidad también fundamental en el reino inteligible, abreviada en las palabras de Marije Martijn: “(...) the nature and the methods of philosophy of nature presuppose a fundamental and crucial continuity between the world of generation and the intelligible realm." ${ }^{57}$ La autora afirma enérgicamente más abajo:

[55] Cfr. Proclo, ET, $§ 146$.

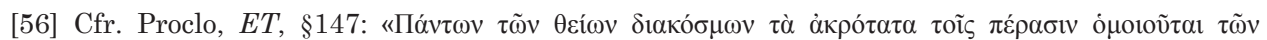

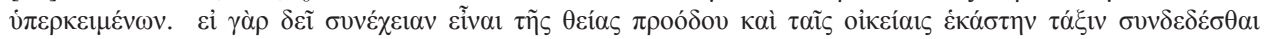

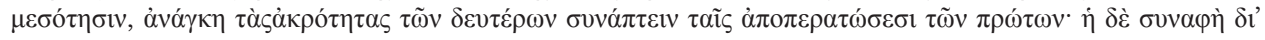

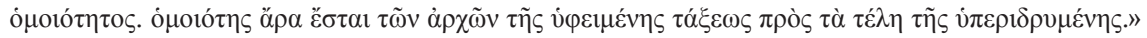

[57] Marije Martijn, "Proclus on nature : philosophy of nature and its methods in Proclus' Com-

THÉMATA. Revista de Filosofía, Nº 49 enero-junio (2014) pp.: 105-125

doi: 10.12795/themata.2014.i49.06 
"I will show, however, that Proclus in his overall reading of the Timaeus is concerned especially with the continuity both of reality and of cognition. All his writings are deeply imbued with the principle "all in all, but appropriately to each thing" ${ }^{58}$. Se puede colegir que no solamente hay una continuidad en lo real, sino también, y profundamente vinculada, una continuidad entre lo real y el reino del pensamiento, como más arriba se puso de manifiesto.

Si bien se habla de Proclo como aprobando la existencia de dioses, esto es, sosteniendo una metafísica politeísta, alejada en algunos aspectos del cristianismo posterior -aunque semejante en otros-, la riqueza de este pensamiento ha contribuido a formar una notable esfera de influencia, en la cual encontramos a Dionisio Areopagita, Juan Scoto Eriúgena y el aporte del Liber de Causis, entre otros.

mentary on Plato's Timaeus", en: Philosophia antiqua, v. 121, Boston: Brill, 2010, p.2. [58] Marije Martijn, "Proclus on nature...", p. 7.

THÉMATA. Revista de Filosofía, No 49 enero-junio (2014) pp.: 105-125

doi: 10.12795/themata.2014.i49.06 


\section{Referencias bibliográficas:}

\section{Obras de Proclo:}

Proclus, Commentary on Plato's Timaeus, Vol. II, Book 2: Proclus on the Causes of the Cosmos and its Creation, traducción, introducción y notas de David T. Runia y Michael Share, Cambridge: Cambridge University Press, 2008. Proclus, De malorum subsistentia (cit. DMS), ed. H. Boese, Berlín: Berolini, 1960.

Proclus, Elementa Theologiae, E. R. Dodds (2nd. ed.), Oxford: Clarendon Press, 1963 (Repr. 1977).

Proclus, In Platonis Cratylum commentaria, ed. G. Pascuali, Lipsiae, 1908. Proclus, In Platonis Timaeum Commentaria, Ed. E. Diehl, 3 vols., Leipzig: Teubneri, 1903-6.

Proclus, Théologie Platonicienne, ed. H. D. Saffrey and L. G. Westerink, 6 vols, Paris: Société d'édition "Les belles lettres", 1968-97.

\section{Bibliografía Secundaria:}

De Haas, "John Philoponus' New Definition of Prime Matter. Aspects of its Background in Neoplatonism and the Ancient Commentary Tradition", Philosophia Antiqua, 69, New York -Köln, 1997.

Dionisio Areopagita, en Los Nombres Divinos, $1^{\circ}$ ed., trad. y notas por Pablo A. Cavallero, revisión y comentarios al texto por Graciela Ritacco, Buenos Aires: Losada, 2007.

Edward P. Butler, The Metaphysics of Polytheism in Proclus, United States: UMI, 2003.

Gabriela Caram, "Recepción y desarrollo del argumento de contigüidad ontológica en el Comentario a las Sentencias de Tomás de Aquino", Estudios Filosóficos, vol. LXII, N 181, Editorial San Esteban, 2013, pp.519-536.

Ineke Sluiter, "Commentaries and the Didactic Tradition", Glenn W. Most (ed.), Commentaries - Kommentare (Aporemata, 4), Göttingen: Vandenhoeck \& Ruprecht, 1999, 173-205.

John Phillips, Order From Disorder. Proclus' Doctrine of Evil and its Roots in Ancient Platonism, Edited by Robert M. Berchman and John F. Finamore, Boston: Brill, 2007.

Los Elementos de Euclides, ed. crítica de M. L. Puertas, Madrid: Gredos, 1996. Marije Martijn, "Proclus on nature: philosophy of nature and its methods in Proclus' Commentary on Plato's Timaeus", Philosophia antiqua, v. 121, Boston: Brill, 2010. 
Platón, Diálogos. IV. Filebo. Timeo. Critias, Introducciones, traducciones y notas de María Ángeles Durán y Francisco Lisi, Madrid: Gredos, 2000. Platón, Diálogos. VIII. Leyes (Libros I-VI), Madrid: Gredos, 1999.

Radek Chlup, Proclus. An introduction, Cambridge: Cambridge University Press, 2012.

S. E. Gersh, "From Iamblicus to Eriugena. An investigation of the Prehistory and Evolution of the Pseudo Dionysian Tradition", Studien zur Problemgeschichte der antiken und mittelalterlichen Philosophie, 8, Leiden: Brill, 1978.

Sarah Klitenic Wear y John Dillon, Dionysius the Areopagite and the Neoplatonist Tradition, England: Ashgate, 2007.

Taylor, "The Philosophy of Proclus", Proceedings of the Aristotelian Society, New Series, Vol. 18, London: Wiley-Blackwel, 1917-1918.

Werner Beierwaltes, Proclo. Ifondamenti della sua metafisica, trad. de Nicoletta Scotti, Introd. de Giovanni Reale, Milano: Pubblicazioni della Università del Sacro Cuore, 1988. 
